\title{
An optical metrology system for the measurement of the refractive index of glass
}

\author{
Inês Leite ${ }^{1,2 *}$, and Alexandre Cabral ${ }^{1,2}$ \\ ${ }^{1}$ Instituto de Astrofísica e Ciências do Espaço, Universidade de Lisboa, Campo Grande 1749-016 Lisboa Portugal \\ ${ }^{2}$ Faculdade de Ciências da Universidade de Lisboa, Departamento de Física, Campo Grande 1749-016 Lisboa Portugal
}

\begin{abstract}
The measurement of the refractive index of parallel plated, optically simple, glass samples is a common and fundamental activity in numerous fields of expertise. This work aimed to optimize a known technique to a simple, cost-effective and reliable system to be implemented in a lab environment, with an accuracy in the results better than $10^{-2}$. A setup with a $632.8 \mathrm{~nm}$ HeNe laser, automatic stage and CMOS camera was used and data was acquired with the help of LabVIEW controlling software. All system components were carefully controlled and optimized with the help of an uncertainty budget. Measurements had an associated uncertainty in the range from $10^{-3}$ to $10^{-4}$.
\end{abstract}

\section{Introduction}

The determination of the refractive index depends on simple concepts of optics [1], and there are several wellestablished methods [2] of measurement of the refractive index (RI) of solid samples. Depending on several factors (type of sample, available equipment, desirable results, and so on) the implemented technique will drastically differ. A state-of-the-art study was conducted on the available methods to implement in order to build a simple, cost-effective and automated system capable of obtaining the refractive index of optically simple samples.

\subsection{Analysis of candidate techniques}

The analysis of several existing techniques was conducted and some key comparisons were distinguished: the method and technique used, the uncertainty obtained in RI measurement, the complexity of the method and the possibility of optimization - that is, the possibility of altering and optimizing an already existing technique in order to fulfil the set objectives. Based on desired system characteristics, two main methods were considered: Lateral displacement and interferometry.

\subsubsection{Lateral displacement vs interferometry}

The lateral displacement method relates an incident light beam that crosses a transparent solid sample to the exiting beam [3]. Typically, the incidence angle is known, as well as the wavelength of the beam - to determine the refractive index of the material, usually the exit angle and/or beam displacement are recorded and through notions of geometry and algebra the refractive index of the material can be known using Eq. 1 .

$$
n_{\text {glass }}=\sqrt{\left(\frac{\cos \theta_{i}}{1-\frac{d}{t \sin \theta_{i}}}\right)^{2}+\sin ^{2} \theta_{i}} \cdot n_{\text {air }}
$$

Another method for determining the RI of a sample is to employ a simple Michelson interferometer to measure the difference in optical path length created by rotating the glass plate located in one of the arms. Considering a similar setup as the one illustrated in Fig. 1, and replacing the camera with the mirror measurement arm, the refractive index can also be determined through a specific equation [4].

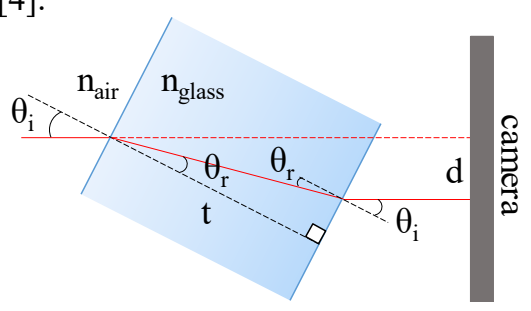

Fig. 1. Lateral displacement $d$, in a camera, of the beam with incident angle $\theta_{i}$, by a glass sample of thickness $t$.

After building a theoretical model of the performance of both techniques and conducting simple experimentation in a setup with similar conditions it was determined that, while in theory the interferometer, with its higher sensitivity and accuracy is capable of providing results with much better uncertainty, due to its relative measurement principle and instability, experimentally, it proved more complex to implement, which critically deviated from the objectives. The lateral displacement

\footnotetext{
*Corresponding author: ines.leite995@gmail.com
} 
method, a technique capable of providing absolute measurements, observed to be insensitive to vibrations and of easier implementation, was selected instead.

\section{Refractive index of glass by optimized lateral displacement}

The final setup of the system is displayed in Fig. 2. The working principle of the system is as follows: The beam that exists the laser will perpendicularly cross the parallel plated sample mounted in the sample holder and a spot image, the zero position, will be captured by the $2 \mathrm{D}$ sensor, the camera. The sample holder, mounted on the automatic stage will then rotate symmetrically in the $y$ axis to both sides of the perpendicular position and the controlling software will record the spot position in every rotation step, performing one measurement cycle.

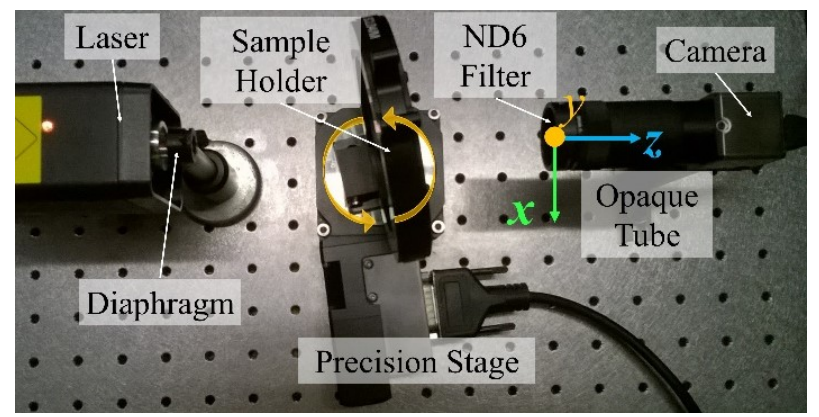

Fig. 2. Final system setup with an $\mathrm{HeNe}$ laser $(632.8 \mathrm{~nm})$, automatic 1-axis rotation stage and CMOS camera.

It was theorized, and later experimentally verified, that the value of the RI becomes unstable as the incident beam nears the perpendicular position to the sample surface, therefore more measurements statistically improve the average of the obtained results. For every position, the spot centre $x y$ coordinates are determined, which in turn are associated to a specific incidence angle, $\theta_{i}$. The deviation from the zero position, lateral displacement $d$, is calculated and by using Eq. 1 and knowing the sample thickness, $t$, multiple values of the refractive index, for the same sample, are obtained.

\subsection{Uncertainty budget}

A useful approach, that greatly benefits the effort to optimize the system, is the construction of an uncertainty budget that encompasses every parameter capable of affecting the uncertainty of the refractive index of glass. Each parameter can have more than one source of uncertainty associated to it, and each source often has a different weight in the final uncertainty of each parameter. Fig. 3 gives a global overview of the main parameters, $d$, $\theta_{i}$ and $t$, as well as each source origin and weight contribution to the uncertainty in RI measurements.

\subsection{Results}

By carefully monitoring the sources of uncertainty, the system was now ready to be validated. Two known

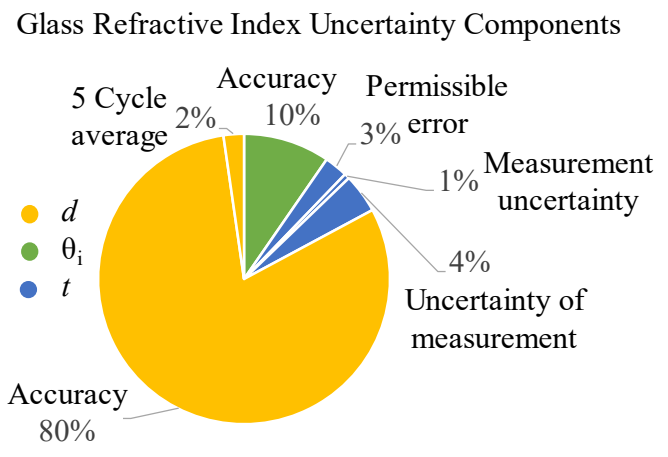

Fig. 3. Uncertainty budget of the built system and every contribution the measurements uncertainty by the three main parameters $d, \theta_{i}$ and $t$.

calibrated samples, N-BK7 and UVFS, were first used to assess the performance of the system and its reliability. Obtained results achieved an uncertainty in the order of $10^{-4}$ for calibrated samples and $10^{-3}$ by two other known samples, of inferior optical quality, as displayed in Table 1.

Table 1. System results of measurements of the RI to four samples: N-BK7 and UVFS (calibration) and B270 glass and a piece of a block of acrylic.

\begin{tabular}{c|ccccc} 
Sample & Exp. RI & Meas. RI & $\pm \mathbf{U}$ & $\begin{array}{c}\mathbf{U} \\
(\mathbf{\%})\end{array}$ & $\begin{array}{c}\mid \text { error } \\
(\mathbf{\%})\end{array}$ \\
\hline N-BK7 & 1.5151 & 1.5151 & 0.0012 & 0.08 & $<0.01$ \\
UVFS & 1.4570 & 1.4571 & 0.0010 & 0.07 & 0.01 \\
B270 & 1.5211 & 1.5219 & 0.0052 & 0.34 & 0.05 \\
Acrylic & 1.4890 & 1.4887 & 0.0016 & 0.11 & 0.02
\end{tabular}

The system works and is ready to be used by the lab, but improvements can always be made. Throughout the work, an interference pattern could not be mitigated due to the nature of the coherent light source - its substitution by an incoherent light source should improve measurement accuracy of the images captured by the CMOS camera, linked to the lateral displacement, the parameter with the greatest contribution to the RI uncertainty.

This work was supported by Fundação para a Ciência e a Tecnologia (FCT) through the research grants UIDB/044 $34 / 2020$ and UIDP/04434/2020 and the participation was supported by Sociedade Portuguesa de Óptica e Fotónica (SPOF).

\section{References}

1. H. Bach, N. Neuroth, The Properties of Optical Glass (Springer-Verlag Berlin Heidelberg 1998)

2. S. Singh, Phys. Scr. 65, 167-180 (2001)

3. V. Kolchinskiy, C. H. Shih, I. Lo, R. Romashko, Phys. Proc. 86, 176-180 (2017)

4. I. Leite, A. Cabral, Proc. SPIE 11207 (2019) 